\title{
Cognitive changes in conjunctive rule-based category learning: An ERP approach
}

\author{
Rahel Rabi ${ }^{1}$ - Marc F. Joanisse ${ }^{2} \cdot$ Tianshu Zhu ${ }^{2} \cdot$ John Paul Minda ${ }^{2}$
}

Published online: 25 June 2018

(C) Psychonomic Society, Inc. 2018

\begin{abstract}
When learning rule-based categories, sufficient cognitive resources are needed to test hypotheses, maintain the currently active rule in working memory, update rules after feedback, and to select a new rule if necessary. Prior research has demonstrated that conjunctive rules are more complex than unidimensional rules and place greater demands on executive functions like working memory. In our study, event-related potentials (ERPs) were recorded while participants performed a conjunctive rule-based category learning task with trialby-trial feedback. In line with prior research, correct categorization responses resulted in a larger stimulus-locked late positive complex compared to incorrect responses, possibly indexing the updating of rule information in memory. Incorrect trials elicited a pronounced feedback-locked P300 elicited which suggested a disconnect between perception, and the rule-based strategy. We also examined the differential processing of stimuli that were able to be correctly classified by the suboptimal single-dimensional rule ("easy" stimuli) versus those that could only be correctly classified by the optimal, conjunctive rule ("difficult" stimuli). Among strong learners, a larger, late positive slow wave emerged for difficult compared with easy stimuli, suggesting differential processing of category items even though strong learners performed well on the conjunctive category set. Overall, the findings suggest that ERP combined with computational modelling can be used to better understand the cognitive processes involved in rule-based category learning.
\end{abstract}

Keywords Category learning $\cdot$ Event-related potentials $\cdot$ Rule learning $\cdot$ Computational modeling

Categorization is a core cognitive process that we rely on constantly to help organize the world around us. Individuals continually acquire new categories through the process of hypothesis testing, where rules are formulated and tested to determine whether they can be used to determine category membership. The process of hypothesis testing encompasses several key cognitive abilities, such as working memory, selective attention, inhibitory control, and other executive functions. In learning explicit rules, individuals must generate hypotheses regarding the possible rule(s), maintain candidate rules in working memory, switch between rules, and update this information in working memory. During this hypothesis testing process, the kinds of categorization rules that the learner formulates and uses can vary in complexity. For example, younger children might find it easy to learn to categorize shapes by

John Paul Minda

jpminda@uwo.ca

1 The Rotman Research Institute of Baycrest Centre, Toronto, Canada

2 Department of Psychology \& Brain and Mind Institute, The University of Western Ontario, London, ON N6A 5C2, Canada using a simple, single-dimensional rule, such as the number of sides the shape has. Older children may be able to learn to use a conjunctive two-dimensional rule to categorize shapes based on the number of sides and the number and measurements of the angles. Likewise, adults may rely on single-dimensional rules when performing tasks, such as organizing files based on urgency and rely on more two-dimensional rules when performing tasks like doing the laundry (e.g., sorting clothes based on colour and washing procedure) or driving (e.g., determining when to drive based on the colour of the traffic light and the presence of pedestrians).

\section{Fundamental mechanisms involved in category learning}

Behavioural and neuropsychological studies have demonstrated the underlying cognitive mechanisms involved in category learning. For example, executive functions (working memory, inhibitory control, etc.) are thought to be necessary for learning rule-based categories. Decreased working memory capacity can interfere with rule-based category learning in 
individuals with frontal lobe damage (Schnyer et al., 2009), children (Huang-Pollock, Maddox, \& Karalunas, 2011; Minda, Desroches, \& Church, 2008; Rabi, Miles, \& Minda, 2015; Rabi \& Minda, 2014), older adults (Maddox, Pacheco, Reeves, Zhu, \& Schnyer, 2010; Rabi \& Minda, 2016), and cognitively depleted adults (Miles \& Minda, 2011; Minda \& Rabi, 2015). Additionally, category learning studies involving some cognitive interference, such as dual or concurrent tasks, have shown that participants display reduced category learning performance when learning a rule-based category set while performing a concurrent task that interferes with working memory (Miles \& Minda, 2011; Minda et al., 2008; Waldron \& Ashby, 2001).

Moreover, Zeithamova and Maddox (2006) examined whether performing a concurrent task would differentially interfere with learning a simple versus a more complex rulebased category set. Both kinds of rules place demands on working memory (Smith, Patalano, \& Jonides, 1998), but a more complicated rule should place heavier demands on the system. In their study, Zeithamova and Maddox asked participants to learn a category set with a suboptimal, singledimensional solution or an optimal, multi-dimensional solution based on a conjunction (like those in Fig. 1). For the simple, single-dimensional rules, participants needed to formulate a rule based on one dimension while ignoring the other dimension. For the conjunctive rule, participants had to attend to and integrate two different featural dimensions to arrive at the correct rule. Selecting and using the conjunctive rule requires more working memory capacity and more cognitive capacity than selecting and using the single-dimensional rule. Zeithamova and Maddox (2006) demonstrated that both single-dimensional and conjunctive rule-based category learning were disrupted by performing a concurrent task. Participants in the conjunctive condition tended to rely more on single-dimensional rules, rather than conjunctive rules, to make categorization decisions.

Functional neuroimaging studies also have examined the link between executive functioning and rule-based category learning (Ashby \& O’Brien, 2005; Poldrack \& Foerde, 2008; Smith \& Grossman, 2008). The brain regions thought to be involved in working memory and selective attention processes, such as the prefrontal cortex and parietal regions, have been shown to be involved when participants learn rulebased categories (Ashby \& Ennis, 2006; Grossman et al., 2002; Seger \& Miller, 2010). Specifically, Jiang et al. (2007) and Li et al. (2009) showed that the prefrontal cortex conveys the crucial conjunctions between key features in determining category membership. Nomura and Reber (2008) showed that the medial temporal lobe plays a key role in successful rulebased categorization. Other research revealed that successful rule-based categorization is associated with increased activity in the anterior medial temporal lobe (Seger, 2008). Nomura and Reber (2008) suggested that the medial temporal lobe acts
Conjunctive Rule

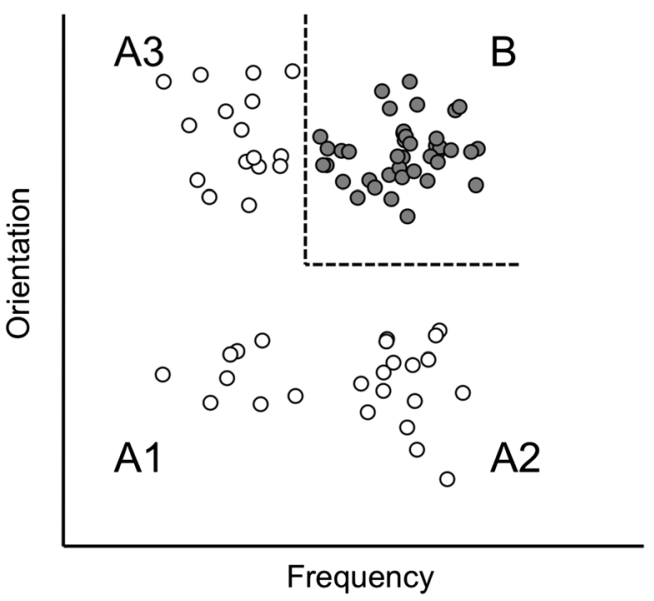

Fig. 1 Conjunctive category structure used. Open circles represent Category A and filled circles represent Category B. The dashed line represents the optimal decision bound

together with the prefrontal cortex structures and the head of the caudate to identify verbalizable rules for categorization.

In line with these findings, Filoteo et al. (2005) showed that differential activation was observed between those participants who learned a single-dimensional, rule-based category set compared with those who did not in frontal and parietal regions which are known to be involved in working memory. Additionally, they also found that the head of the caudate was associated with incorrect responding in participants who successfully learned the rule. The involvement of this structure could either be related to processing negative feedback or initiating a switch between potential category rules. In support of Filoteo et al.' (2005) findings, Monchi et al. (2001) also demonstrated that the caudate is differentially activated on incorrect versus correct trials when participants performed the Wisconsin Card Sorting Test (a task thought to measure rule-based category learning and rule switching). Finally, the basal ganglia interacts with the frontal cortex in corticostriatal loops and exerts some control over the strategy selection in categorization tasks (Seger, 2008). Cincotta and Seger (2007) and Merchant et al. (1997) suggested that the basal ganglia and the corticostriatal loop play a critical part in learning tasks that involves trial and error. Differential activation was seen in corticostriatal loops in subjects' progress from novices to experts in categorization. The anterior caudate determines the rate of learning; greater activation is associated with more rapid learning (Williams \& Eskandar, 2006) and greater sensitivity to prediction error (Haruno \& Kawato, 2006).

Research involving event-related potentials (ERPs) may help to further clarify the cognitive processes involved in rule-based category learning. While prior ERP research on categorization has been limited, some scientists have begun to examine the ERP correlates of rule-based category learning. Because of their high temporal resolution, a large number of 
time-locked categorization trials can be averaged together, allowing researchers to probe cognitive processing with high/millisecond precision. As such, ERPs are well suited to study how participants process stimuli that follow the rule, how they process stimuli that do not follow the rule, and how participants react to feedback. For example, Folstein and van Petten (2011) collected ERP data from participants in a multi-dimensional, rule-based category learning task to analyze the difference in frontal-parietal waves when participants were classifying stimuli that were near the category boundary versus stimuli that were far from the boundary. Near boundary stimuli are less typical and more difficult to classify, because they possess features that are common in the opposing category. Folstein and van Petten found that near boundary stimuli that also possessed features from the opposite category elicited larger ERPs from prefrontal regions compared with nonconflicting stimuli. Larger, positive potentials also were observed for far from boundary stimuli after the peak of parietal P300, perhaps reflecting a different, secondary strategy for the near versus far from boundary stimuli.

More recent research by Morrison et al. (2015) monitored brain activity using ERPs as participants learned, via feedback, to sort Gabor patches that varied in spatial frequency and orientation. Morrison and colleagues used a single-dimensional, rule-based category set, with the rule depending on the frequency of the bands in the Gabor patches. Furthermore, participants had to test various rules, inhibit the incorrect rule corresponding to the orientation dimension, and update this information in memory. ERP findings revealed a differential correct/incorrect response in positive parietal potentials only, with participants displaying a larger correct/incorrect difference performing the task more accurately. These positive potentials were thought to represent a Late Positive Complex (LPC). The LPC is a positive amplitude that peaks after 400 $\mathrm{ms}$ at centroparietal regions and in a category learning task, the LPC corresponds to comparing a stimulus to other category members in memory or category decision evaluation (Craddock, Milham, \& LaConte, 2013; Schendan \& Lucia, 2010). This waveform is likely to be composed of multiple components, such as the modality independent, responselocked, positive-choice response, and the late slow wave.

The LPC is also similar to the P300 in many ways (Dien, Spencer, \& Donchin, 2004; Kok, 2001; Polich, 2007; Rushby $\&$ Barry, 2007). Morrison and colleagues suggested that the differential LPC responses for correct/incorrect responding reflected the engagement of the neural system responsible for making rule-based decisions. With regards to feedback processing, Morrison and colleagues observed a P300 response on incorrect trials. The P300 is an ERP component elicited when a participant responds to a rare target stimulus in decision making tasks that involve attention and short-term memory (Polich, 2007). During the rule-based categorization task participants are forming hypotheses about the rule to use, and when those expectations are violated by negative feedback, participants have to reevaluate their choice of strategy. Morrison and colleagues also considered the alternative conclusion that the P300 effect may reflect memory updating. According to this viewpoint, the rule maintained in memory must be changed as a result of negative feedback.

\section{Rationale for the present research}

Although there is considerable evidence for the role of working memory and other executive functions in rule-based category learning, only a small number of studies have examined this relationship with ERP techniques. The previously described studies asked participants to learn a single-dimensional rule but almost nothing is known about how these results will generalize to more complicated, two-dimensional rules. The current study will examine the event-related brain potential correlates of complex, conjunctive rule-based category learning. In line with the findings of Morrison et al., we predict that a differential correct/incorrect response will be found in positive parietal potentials, indicative of an LPC. The LPC is thought to be an index of memory access and updating (Polich, 2007) and correct responses should result in a larger LPC compared to incorrect responses, representing the updating of rule information in memory. Also, in line with Morrison et al., we predict a larger P300 for incorrect compared to correct trials. The P300 is an index of violations of expectancy and so incorrect responses should influence a participant's confidence in the explicit rule they are choosing to use.

The current study will use what is known about ERP signatures of category learning to identify the mechanisms by which individuals converge on the correct categorization rule. Compared with the single-dimensional, rule-based task used by Morrison et al. (2015), the use of a more complex conjunctive rule-based task in the current study will enable us to examine a wider range of ERP effects. In addition to examining the ERPs associated with stimulus and feedback processing, the present study also will allow us to look at how specific stimuli are processed differently based on their predicted classification according to one of two easier, single-dimensional, suboptimal rules or a more difficult, two-dimensional, optimal rule. While on the surface it may appear that a participant has learned and correctly applied a conjunctive rule, ERP measures may reveal that the participant processes individual stimuli differently based on features they possess (i.e., whether a stimulus belonging to one category set shares a dimensional value with stimuli belonging to another category set). The ERP data therefore can provide valuable insights into the process of category learning that are not available strictly through analyses of behavioural (accuracy and reaction time) data. 
Finally, the present study examined the fit of several computational models to the behavioral data at successive points in the category learning trajectory to understand how strategies may change with time (Filoteo, Lauritzen, \& Maddox, 2010; Nadler, Rabi, \& Minda, 2010; Rabi \& Minda, 2017). This is particularly informative for the conjunctive rule category set, because we predict that participants will first acquire the single-dimensional, suboptimal rules and will then shift to the more complicated and optimal conjunctive rule. This transition may not occur for all participants and is expected to rely on executive function abilities like working memory.

\section{Methods}

\section{Participants}

Thirty-eight undergraduate students were recruited from the University of Western Ontario. The data from three participants were not included in the analyses, because two of them performed at chance on the categorization task and the third participant had excessive ocular artifacts, leaving 35 participants in the final analyses (14 males and 21 females; mean age $=20.10$ years, $S D=3.37$ ). All participants reported normal or corrected to normal vision and fluency in English. Participants received either course credit or $\$ 20$ for their participation in the study.

\section{Materials}

The category structure used in this study was formally identical to the category structure used in the Zeithamova and Maddox (2006) study, although we generated a unique set of exemplars for our study. For the category learning task, participants classified sine-wave gratings that varied in spatial frequency and orientation. There were 40 Category A and 40 Category B stimuli. The 80 stimuli were generated by sampling from 4 bivariate normal distributions. Three of these distributions were assigned to Category $\mathrm{A}$ and one to Category B. The distribution parameters that define each of the distributions are displayed in Table 1. A scatterplot of how

Table 1 Distribution parameters for the conjunctive rule-based category set

\begin{tabular}{llllll}
\hline Category structure & $\mu_{\mathrm{f}}$ & $\mu_{\mathrm{o}}$ & $\sigma^{2}$ & $\operatorname{cov}_{\mathrm{f}, \mathrm{o}}$ & $\mathrm{N}$ \\
\hline Category $\mathrm{A}_{1}$ & 283 & 98 & 75 & 0 & 8 \\
Category A $_{2}$ & 317 & 98 & 75 & 0 & 16 \\
Category A & 283 & 152 & 75 & 0 & 16 \\
Category B & 317 & 152 & 75 & 0 & 40 \\
\hline
\end{tabular}

Stimuli from the $A_{1}, A_{2}$, and $A_{3}$ distributions were all members of Category A the stimuli are represented in two-dimensional space, and the optimal rule that can correctly classify all the exemplars is presented in Figure 1. Participants should respond Category $\mathrm{B}$ when the spatial frequency was high and the orientation was steep, and they should respond Category A otherwise. We then generated sine-wave gratings corresponding to each coordinate sampled from the distributions above. For both category sets, sine-wave grating frequency was calculated as $f=$ $0.25+\left(x_{\mathrm{f}} / 50\right)$ cycles per stimulus and orientation was calculated as $o=x_{o} \times(\pi / 20)$ degrees. The stimuli and the $\mathrm{R}$ code needed to create the category set are available at http://osf.io/ $89 \mathrm{~g} 6 \mathrm{~m} /$.

\section{Procedure}

Category learning task In the category learning task, participants were told that they would be seeing an image on the screen and their job was to determine whether that image belonged to Category A or Category B. Responses were made using a button box labeled "A" and "B" for Categories A and $\mathrm{B}$, respectively. Participants were told that they would receive feedback after every response and that they should use this feedback to help them learn to make as many correct responses as possible. Stimuli were presented electronically using E-Prime 2.0 software (Psychology Software Tools, 2012) via a 17 -inch CRT monitor.

The manner in which the stimuli were presented on each trial is displayed in Figure 2. On each trial, participants saw a fixation cross, followed by a sine-wave grating in the center of the screen and an A and B in the upper left and upper right corner of the screen. Upon making a response, the sine-wave grating disappeared, and feedback was delivered in the center of the screen (either a checkmark or an X). If a participant took longer than 2,500 ms to respond, no feedback was presented, and no response was recorded for that trial. Between each trial, a blank screen appeared for $750 \mathrm{~ms}$. Participants were presented with 6 blocks of the 80 stimuli; 480 trials in total. Within a block, the order of presentation of all 80 stimuli was randomized for each participant.

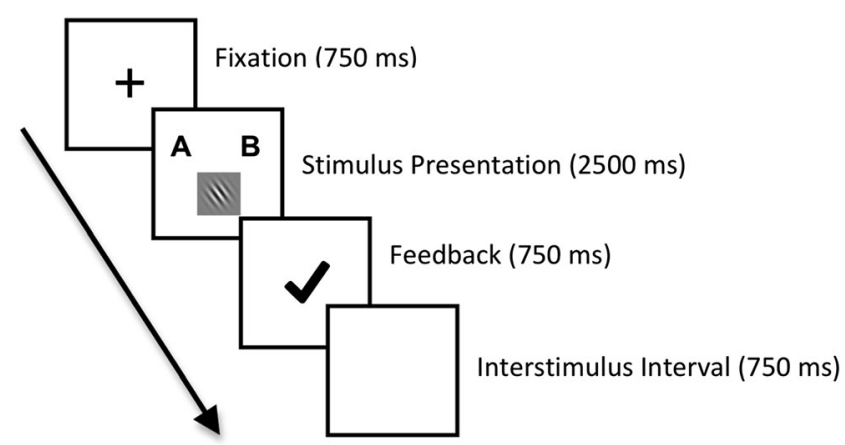

Fig. 2 Stimulus presentation during each trial 
Participants completed five practice trials to familiarize themselves with the task before data collection, and there was a short break (approximately 20 seconds) between each block. Participants were warned in advance that they would have 2.5 seconds to make a response, resulting in very few "no response" trials. Participants also were advised to blink during the blank screen following feedback to avoid excessive blinking during stimulus and feedback presentation which would contaminate EEG recordings.

EEG recording and preprocessing EEG data were recorded at 32 scalp sites placed in the international 10-20 orientation using BioSemi ActiveTwo $\mathrm{Ag} / \mathrm{AgCl}$ electrodes embedded in a custom elastic cap (BioSemi, Amsterdam, The Netherlands). Electro-oculogram (EOG) activity was recorded from active electrodes placed above, beside, and beneath the left eye, and beside the right eye. An additional active electrode (CMS common mode sense) and a passive electrode (DRL - driven right leg) were used to comprise a feedback loop for amplifier reference. Two additional electrodes were placed at the left and right mastoids for offline re-reference. All EEG electrode impedances were maintained below $20 \mathrm{k} \Omega$. All bioelectric signals were digitized on a PC using ActiView software (BioSemi) at a rate of $512 \mathrm{~Hz}$ with a bandpass of $0.1-100 \mathrm{~Hz}$ and a $60 \mathrm{~Hz}$ notch filter.

Offline analysis was performed using EEGLAB v. 13.4.3b (Delorme \& Makeig, 2004) and ERPLAB v. 4.0.3.1 (LopezCalderon \& Luck, 2014). All data were re-referenced to the mean left/right mastoid electrodes, and bandpass filtered with cutoffs of $0.1 \mathrm{~Hz}$ and $30 \mathrm{~Hz}$. The trials were epoched from 200 $\mathrm{ms}$ before the onset of the target stimulus to $800 \mathrm{~ms}$ after the onset of the target stimulus, and baseline corrected to the 200ms prestimulus onset. The data were segmented into stimuluslocked and feedback-locked epochs. Trials containing eye blinks and other nonocular artifacts (EEG activity exceeding \pm 75 microvolts at any electrode) were discarded. No more than $25 \%$ of trials were rejected for any subject.

\section{Results}

The data category learning data, raw EEG data, and the R code used to conduct the computational modeling can be found at http://osf.io/89g6m/.

\section{Behavioural analyses}

Category learning The average proportion correct was obtained by calculating the mean proportion correct in each block for each participant and then averaging across participants. The resulting learning curve is shown in Figure 3. A repeated measures ANOVA revealed a main effect of block, $F$ (3.7, $126.14)=37.99, p<0.001, \eta_{\text {partial }}^{2}=0.53$, [Greenhouse-

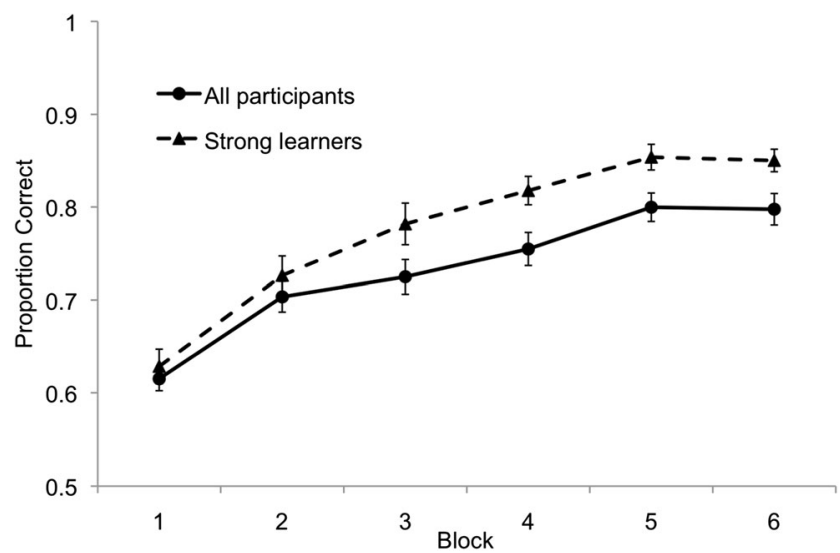

Fig. 3 Average proportion of correct categorization responses as a function of learning block for all participants compared to just the strong learners. Error bars denote the standard error of the mean

Geisser corrected], indicating that learning occurred across blocks. The categorization performance of participants began at $60 \%$ correct during the first block and ended with a categorization performance of $80 \%$ by the final block. For certain ERP analyses, only strong learners were considered.

Strong learners were classified as participants whose response pattern over the last three learning blocks was best fit by the optimal two-dimensional, conjunctive rule model (see below). This group consisted of 20 participants, whose performance began at $63 \%$ during the first block, and ended with a categorization performance of $85 \%$ by the final block (Figure 3).

Computational modeling For insight into the response strategies used by our participants, we fit several classes of decision boundary models to each block of each participant's data (for additional details see Ashby, 1992; Filoteo et al., 2010; Maddox \& Ashby, 1993; Rabi \& Minda, 2017; Zeithamova $\&$ Maddox, 2006). Two unidimensional models were fit to each observer's responses. These models assume that the participant sets a criterion based on one of the stimulus dimensions, either the frequency or orientation of the lines. In both unidimensional models, the intercept of the decision bound was allowed to vary. The varying intercept corresponds to the placement of the decision criteria by the participants. We also fit a class of two-dimensional models to the data which assume that the participant based their categorization decision on both dimensions (i.e., the optimal, conjunctive rule). In one version of this model, the slope and intercept were allowed to vary, which corresponds to an information-integration strategy. In another version of the model, only the intercepts of the two dimensional boundaries were allowed to vary. This second model was a strict version of a conjunctive rule and was the optimal rule for classifying these stimuli. Finally, we fit two guessing models, which assumed no dimensional strategy (one assumed that participants randomly responded A or B with equal probability for each response and the other assumed unequal probability). 
We fit these models to each participant's data by maximizing the log likelihood. Parameters for each model were estimated using the maximum likelihood method, and the relative fit of the models were compared using the Bayesian Information Criterion $(B I C$, where $B I C=\mathrm{r} \ln (N)-2 \ln L ; \mathrm{r}$ is the number of free parameters, $N$ is the number of trials being fit, and $L$ is the likelihood of the model given the data). $B I C$ is a measure of goodness of fit, which penalizes a model for extra free parameters. To find the best model to account for each participant's responses, a $B I C$ value is computed for each model, and the model associated with the smallest $B I C$ value is chosen. A learner was classified as a participant using one of the two strategies (unidimensional, two-dimensional), aside from guessing. The optimal conjunctive rule yields the highest accuracy (close to $100 \%$ ). Note that applying a unidimensional strategy also could result in good performance (i.e., accuracy of up to $80 \%$ ), although this performance would not be as high as those using the optimal strategy. We assumed that both single-dimensional and two-dimensional strategy users would rely on hypothesis testing to test different rules and would rely on working memory to update information based on the feedback received. A specific example of the block-by-block strategy analysis for one participant who transitioned from a single-dimensional strategy to an optimal, conjunctive strategy is shown in Figure 4.

In the strategy analysis, learners were classified as anyone fit by one of the two single-dimensional models (frequency or orientation) or one of the two, two-dimensional models (conjunctive or information integration) in their last block of learning. As mentioned in the Methods section, only two participants were excluded from this analysis, because their strategy performance indicated that they were guessing during their final block. Among the remainder of the participants, 9 were best fit by a unidimensional model (i.e., either a frequency or orientation strategy) and 26 were best fit by a two-dimensional model. See Table 2 for a complete list of the proportion of participants fit by each type of strategy per learning block.

\section{ERP analyses}

The data from the most central 13 electrodes were included in the three primary analyses (Figure 5).
Block 1 : Orientation

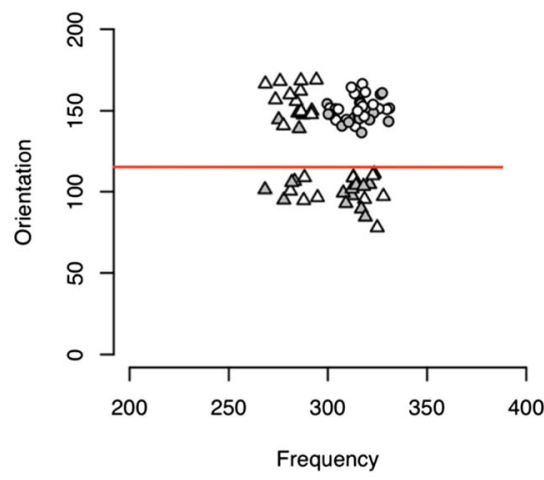

Block 4 : Conjunctive Rule

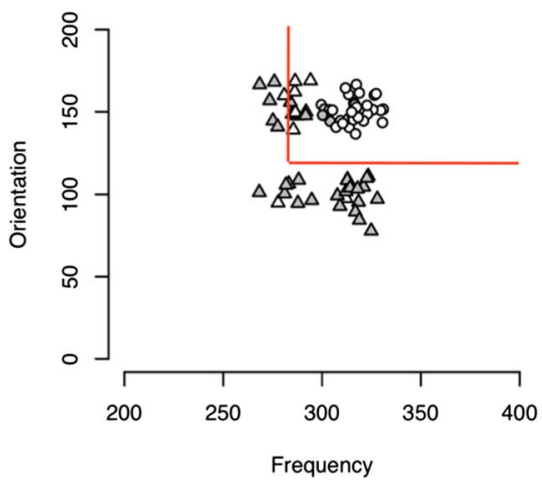

Fig. 4 Example of the modeling results across blocks for one participant's data. Triangles indicate actual Category A items, and circles indicate actual Category B items. Filled symbols indicate a participant's Category A responses, and open symbols indicate a participant's Category B responses. The lines show the best-fitting
Block 2 : Orientation

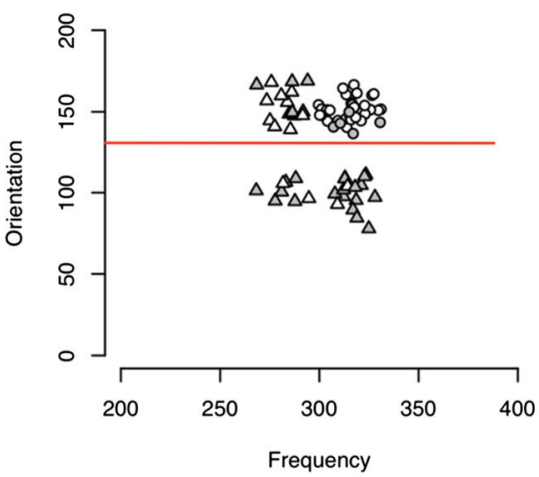

Block 5 : Conjunctive Rule

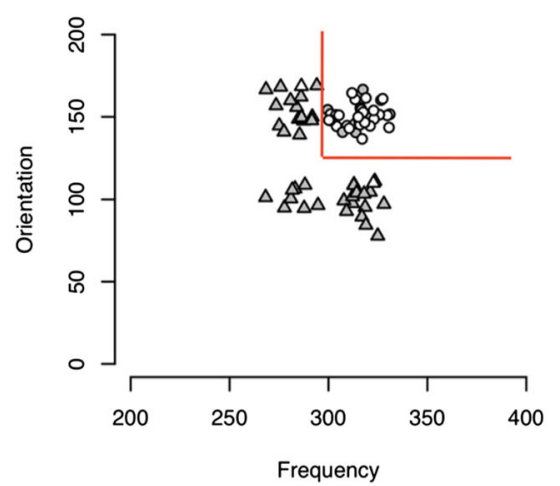

Block 3 : Conjunctive Rule

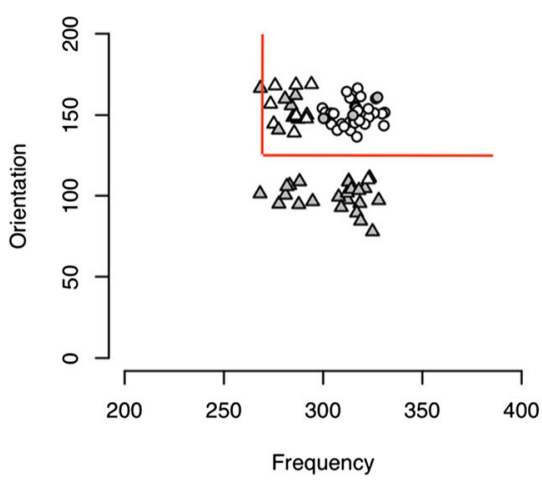

Block 6 : Conjunctive Rule

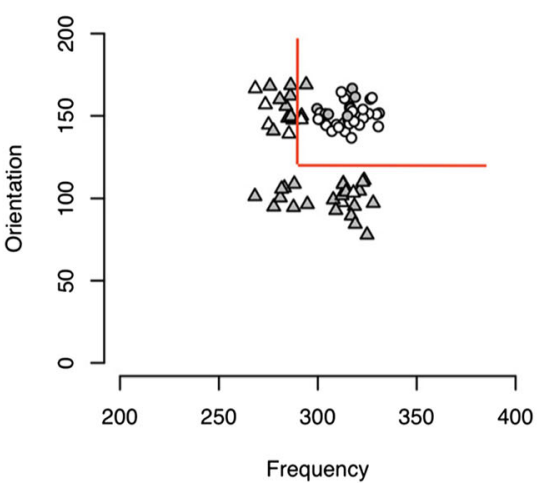

decision-boundary model for each block. In this example, the participant started off using a single dimensional rule (i.e., orientation rule indicated by the horizontal line) but switched to a conjunctive rule for the remainder of the categorization task 
Table 2 Number of subjects fit by each class of decision bound models

\begin{tabular}{llll}
\hline Model & Two-dimensional & Single-dimensional & Guessing \\
\hline Block 1 & 1 & 21 & 13 \\
Block 2 & 10 & 19 & 6 \\
Block 3 & 17 & 12 & 6 \\
Block 4 & 22 & 9 & 4 \\
Block 5 & 28 & 7 & 0 \\
Block 6 & 26 & 9 & 0 \\
\hline
\end{tabular}

The optimal model is shown in bold. Thirty-five participants were included in the study

Category learning Similar to Morrison et al. (2015), correct/ incorrect subtractions were performed to examine category learning. The dependent measure in our analyses was the mean amplitude of the epoch from $300 \mathrm{~ms}$ to $600 \mathrm{~ms}$ poststimulus onset at parietal scalp sites (i.e., the P300 component), P7, P3, Pz, P4, and P8. We averaged the parietal electrodes for the analysis of variance. As shown in Figure 6, a late positive parietal ERP (300-600 ms) was predictive of correct categorization, $F(1,34)=5.03, p=0.03, \eta_{\text {partial }}^{2}=0.128$. The late positive parietal ERP (i.e., late positive component; LPC) was larger for correct $(M=4.56, S D=3.6)$ than incorrect $(M=$ $4.21, S D=3.4$ ) trials, suggesting memory access and updating during category learning. A topographic map of the ERP difference between correct and incorrect trials is illustrated in Figure 7.

Feedback processing ERPs recorded during feedback were examined for the presence of a P300 effect. Correct/incorrect P300 subtractions were performed. The dependent measure in our analyses was the $50 \%$ fractional area latency of the epoch from $300 \mathrm{~ms}$ to $600 \mathrm{~ms}$ after feedback onset at frontocentral, central, and parietal scalp sites, FC1, FC2, C3, C4, Cz, CP1, $\mathrm{CP} 2$, and Pz. We averaged the eight electrodes for the analysis of variance. As expected, a feedback-locked P300 ERP effect was found, $F(1,34)=59.86, p<0.001, \eta_{\text {partial }}^{2}=0.638$, with a pronounced P300 for incorrect $(M=403.55, S D=30.09)$ compared with correct $(M=373.28, \mathrm{SD}=30.07)$ trials (Figure 8).

Note that although the timing of the P300s differed appreciably for correct and incorrect trials, we were primarily interested in the amplitude of this waveform, and not its timing. Thus, the 50\% fractional area latency allowed us to calculate amplitudes in a way that did not require us to set different a priori time analysis windows for the two conditions. The difference in amplitudes suggests that the error feedback response was unexpected for learners. One could speculate that unidimensional learners would be surprised when they made an error, because using a unidimensional rule could still result in good performance if applied consistently. Two-dimensional

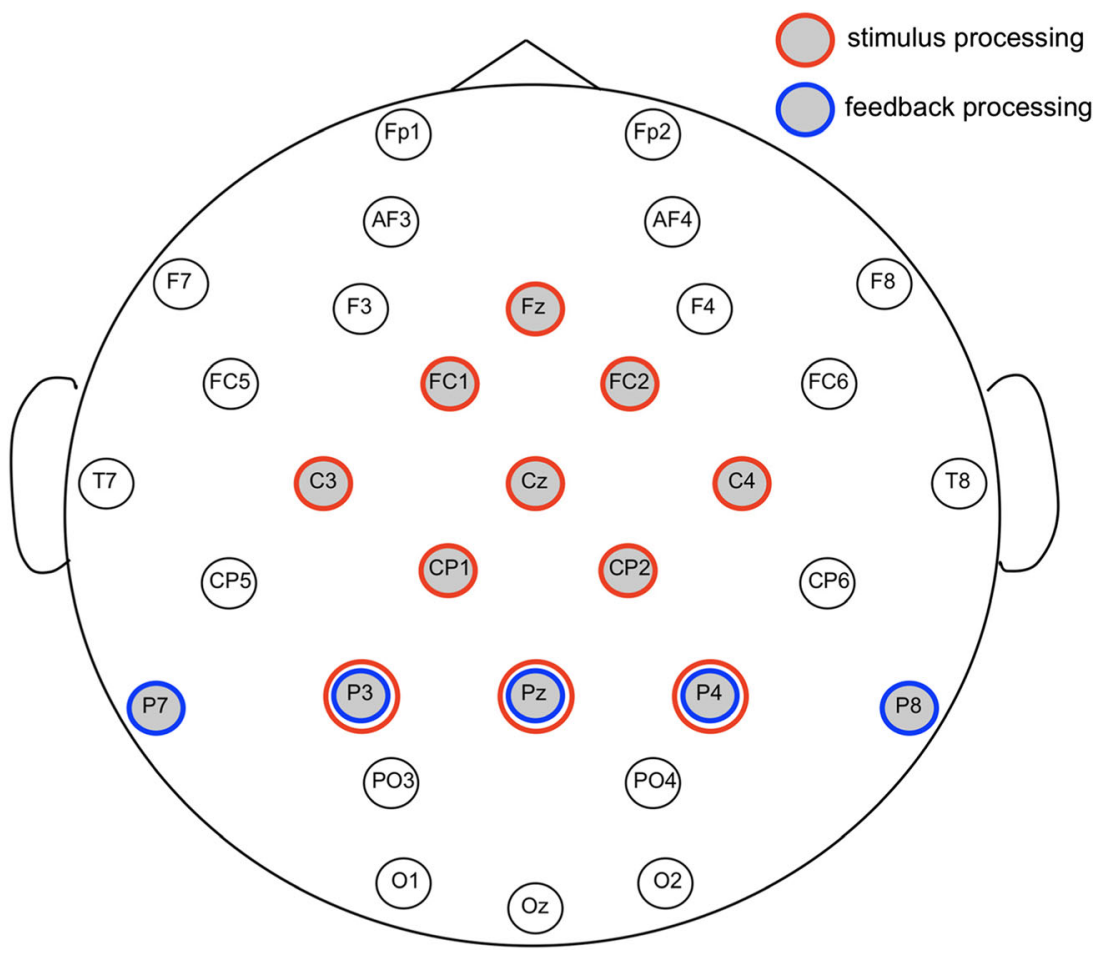

Fig. 5 Electrode montage. Filled circles indicate electrodes included in the analyses. Red outlined circles represent electrodes examined during stimulus processing (i.e., category learning, stimulus difficulty, and stimulus frequency). Blue outlined circles represent electrodes examined during feedback processing 

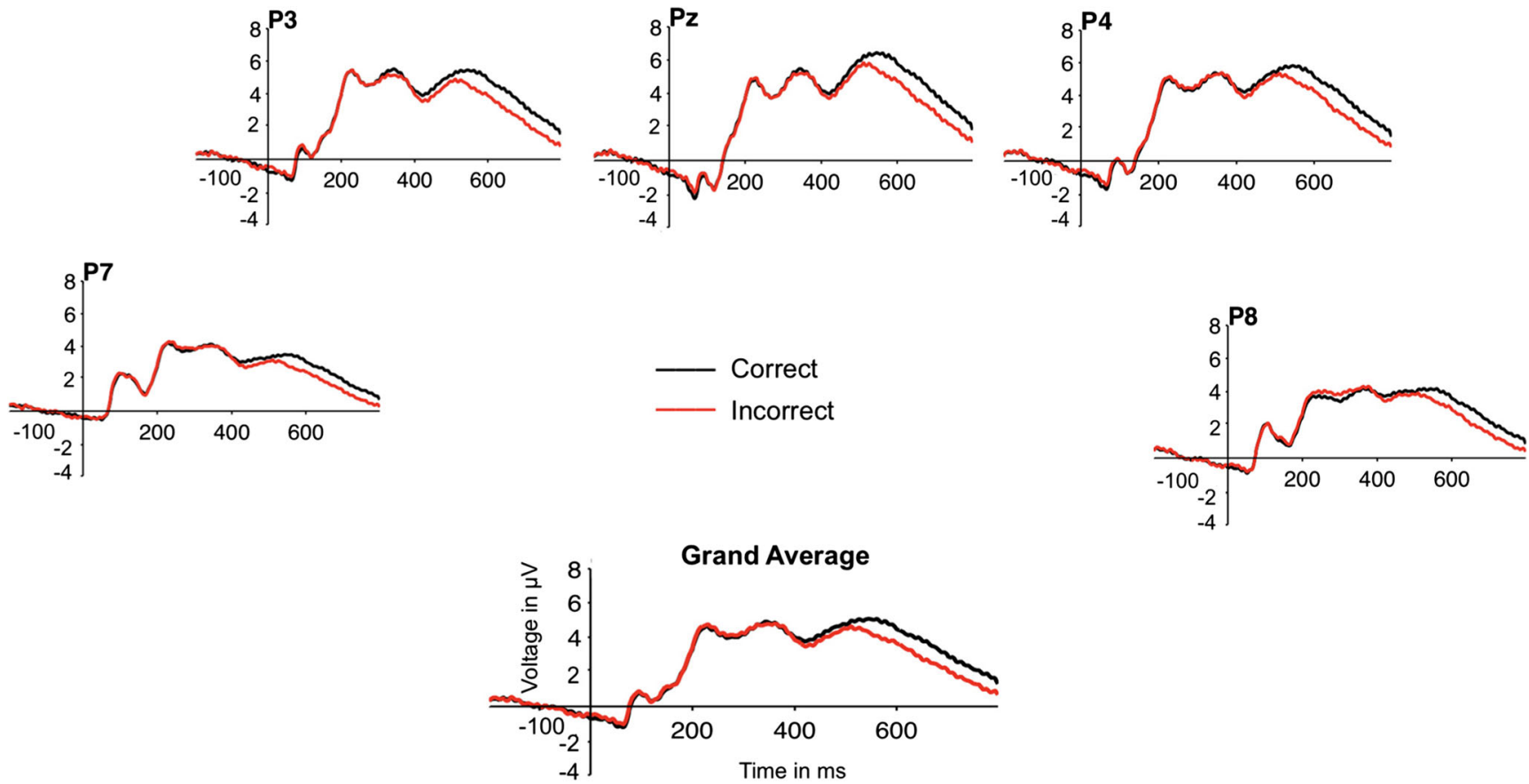

Fig. 6 Stimulus-locked ERPs for correct vs. incorrect trials across parietal electrodes

rule learners also would be surprised when they made an error, because their rule tended to work for the majority of trials.

To confirm that ERP findings related to stimulus processing and feedback processing were not influenced by the inclusion of single-dimensional rule learners, the same analyses mentioned above were conducted excluding the nine singledimensional rule learners. Results remained significant after removing these participants, indicating that singledimensional rule learners did not drive the ERP effects found.

Stimulus difficulty For the purposes of this analysis, an "easy" stimulus is one that can be correctly classified by the suboptimal, single-dimensional strategy as well as the optimal, twodimensional strategy. A "difficult" stimulus is one that would be consistently misclassified by the suboptimal, singledimensional strategy and only correctly classified by the optimal, two-dimensional strategy. We reasoned that even if a participant was correctly using the optimal, two dimensional strategy, they may show differential sensitivity to the difficult stimuli. While on the surface it may appear that strong conjunctive rule-based learners were performing very well, they may have still been processing different types of stimuli differently. For example, the optimal rule required that participants respond Category B when the spatial frequency was high and the orientation was steep and to respond Category A otherwise (which included 3 different subsets of Category A), because there were 40 stimuli belonging to Category B and 40 stimuli belonging to Category A ( 8 stimuli belonging to Category A1, 16 belonging to Category A2, and 16 belonging to Category A3). In this regard, Category B could be interpreted as being the easier category to learn, because there is only one subset of this category and 40 exemplars per learning block and Category B has a stronger family resemblance structure compared with Category A. On the contrary, participants would have to learn that exemplars A1 (low frequency and shallow orientation), A2 (low frequency and steep orientation), and A3 (high frequency and shallow orientation) all belong to Category A, even though they look different. Among the three subtypes of Category A, A2, and A3 were the most difficult to learn, because they shared one dimension

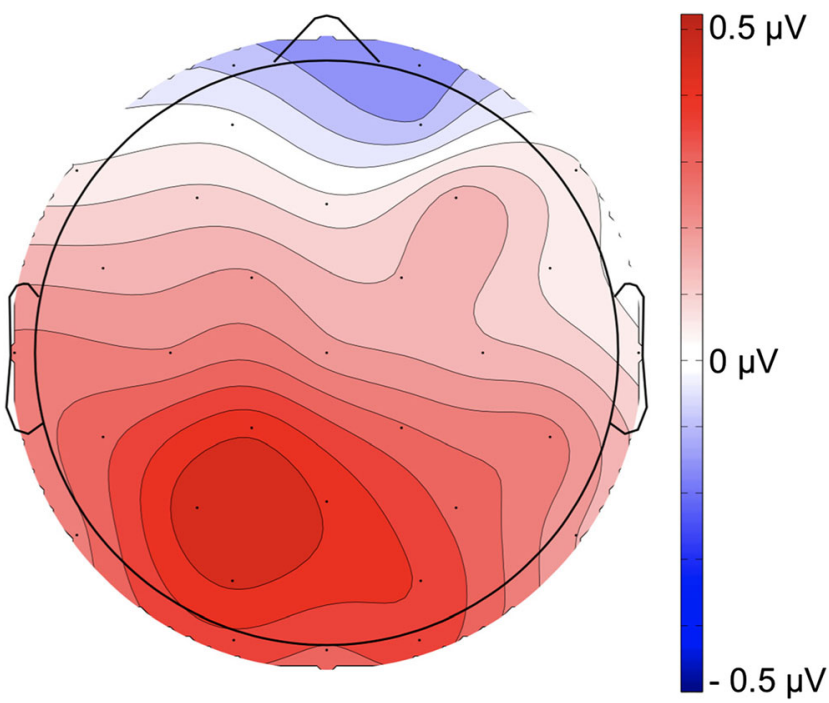

Fig. 7 A topographic map of the correct minus incorrect subtraction of mean amplitude between 300-600 ms for stimulus-locked ERPs 


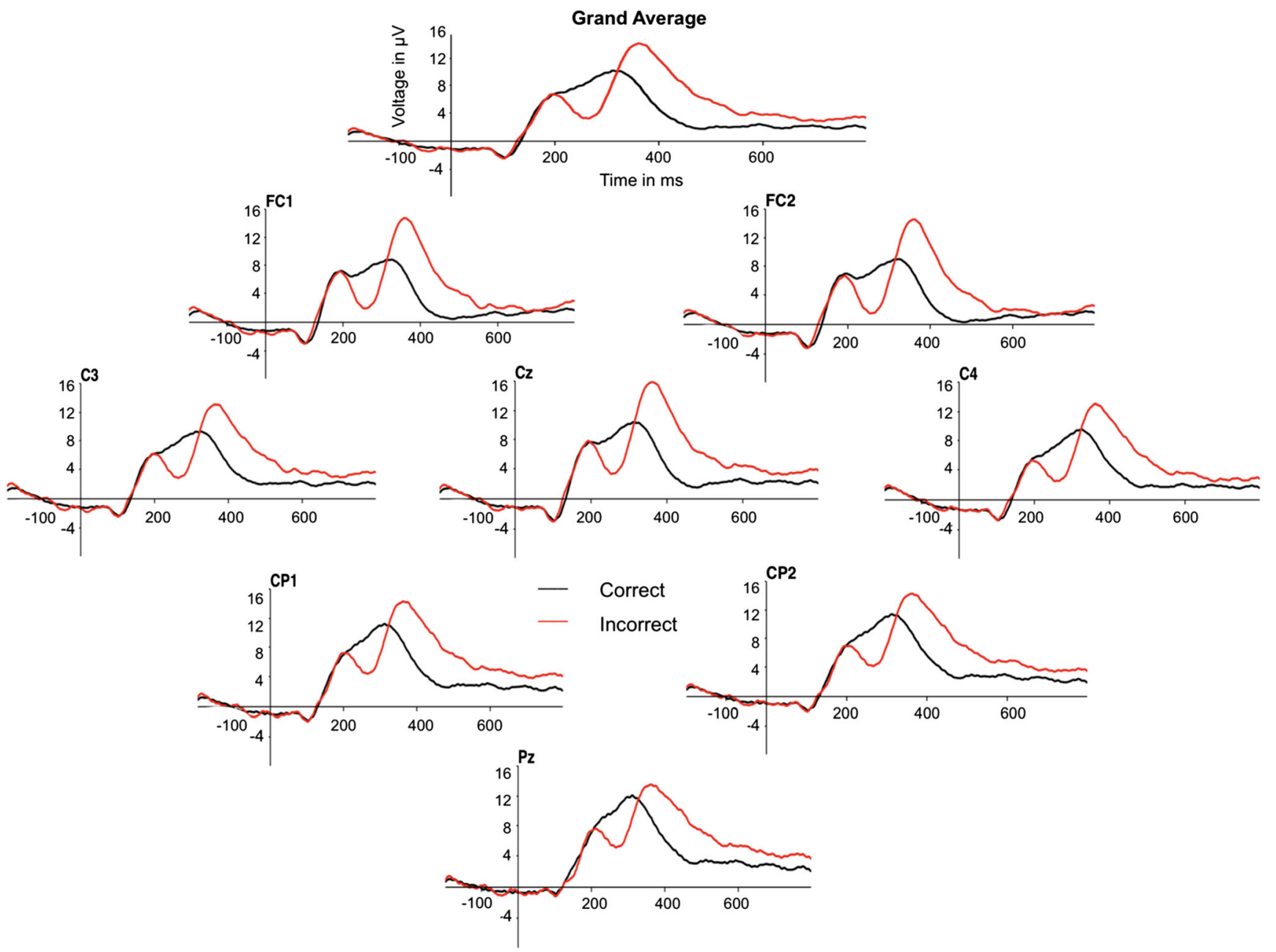

Fig. 8 Feedback-locked ERPs for correct versus incorrect trials

in common with Category B. ERP analyses were used to examine how participants differed in processing easier (Category B) versus difficult (Category A2 and A3) stimuli.

We were particularly interested in strong, conjunctive rulebased learners, because these included individuals who learned a conjunctive rule and consistently applied it across a large number of trials. By analyzing data from strong conjunctive learners, this enabled us to have enough data points to compare correct responses on Category A2/A3 to Category B. A strong learner was classified as any participant who was best fit by the conjunctive, two-dimensional rule during at least the last three learning blocks. There were 20 participants included in this analysis. The dependent measure in our analyses was the mean amplitude of the epoch from $500 \mathrm{~ms}$ to 800 ms post-stimulus onset at parietal and centro-parietal scalp sites, CP1, CP2, P3, Pz, and P4. We averaged the parietal and centro-parietal electrodes for the analysis of variance, for correct responses only.

As shown in Figure 9, a late positive slow wave emerged, $F(1,19)=22.46, p<0.001, \eta_{\text {partial }}^{2}=0.542$, reflecting a larger late positive slow wave for difficult $(M=4.83, S D=3.84)$ compared with easy $(M=3.49, S D=2.89)$ categories. These findings are in line with past research showing that positive slow waves with centro-parietal distributions were elicited by target stimuli in difficult perceptual discrimination tasks (Ruchkin, Johnson, Mahaffey, \& Sutton, 1988; Ruchkin \& Sutton, 1983). Furthermore, research by Gunter, Jackson, and Mulder (1995) suggests that positive slow waves reflect difficulty of perceptual operations and memory storage. Processing Category A2 and A3 stimuli require greater effort and memory requirements than processing exemplars from Category B. To further illustrate this finding, topographic maps of the ERP difference between hard versus easy stimuli, for correct responses only, is illustrated in Figure $10 .^{1}$

Model-based ERP analysis Our computational modelling provided an opportunity to examine the ERP signals associated with the different strategies and potentially to make some inferences about the psychological processes involved in each strategy and the processes involved in shifting from one

\footnotetext{
${ }^{1}$ This analysis was suggested by a reviewer on the original submission.
} 

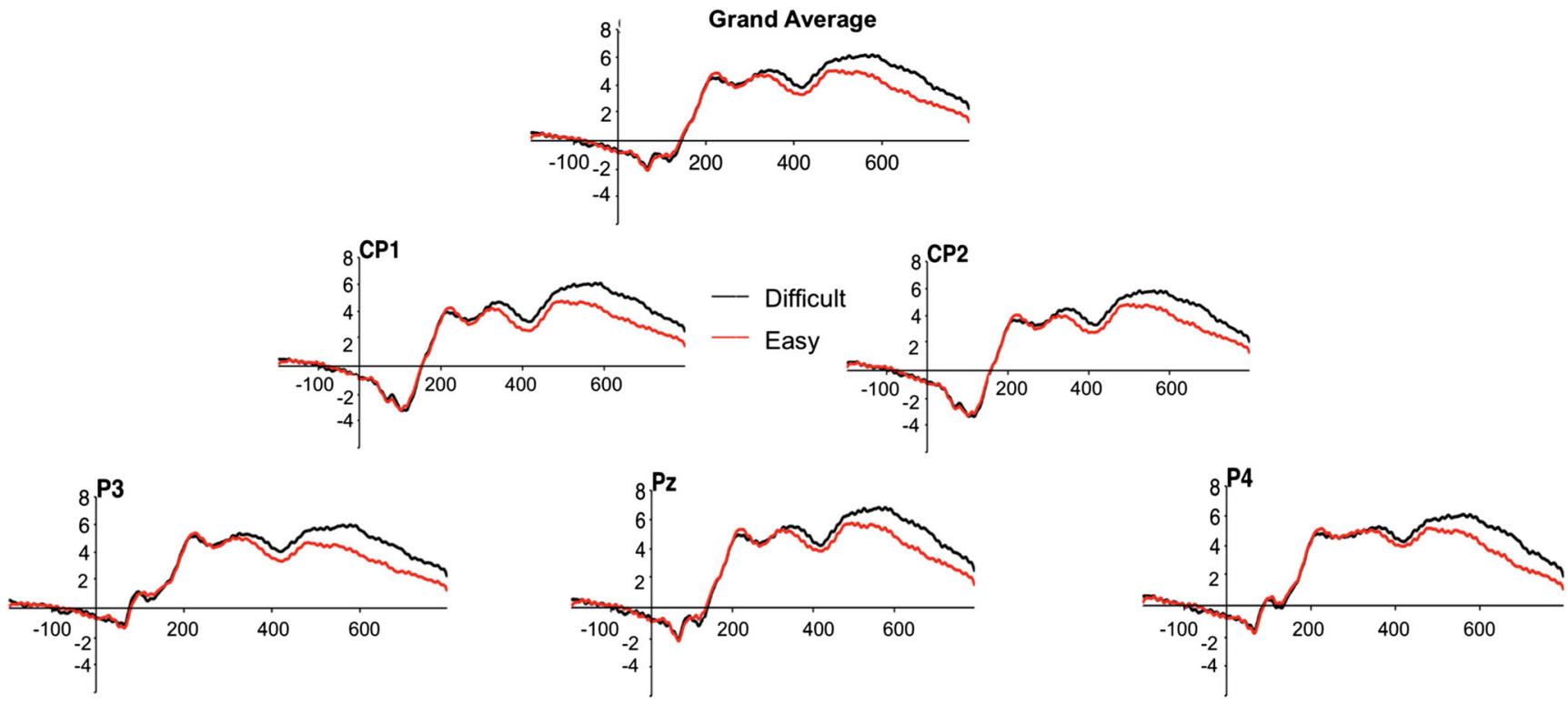

Fig. 9 Stimulus-locked ERPs for "easy" versus "difficult" stimulus items

strategy to another. Among the 20 strong learners, all but two showed evidence of a transition from strong, singledimensional performance to strong, two-dimensional performance. Because this analysis was exploratory, we did not have a specific hypothesis that we were testing. Rather, based on the cognitive psychological demands of the category learning task, we reasoned that we should observe a differential waveform pattern within subjects based on whether they were responding with a suboptimal single dimensional strategy or the optimal, two-dimensional strategy. We selected the frontal region as the ROI, given the role that frontal regions likely play in the hypothesis testing process and the transition to the complex rule.

For this analysis, we examined the performance of the 20 strong learners and removed 2 participants who never showed any evidence of single-dimensional strategies. This left us with 18 participants who displayed both single- and twodimensional strategies during learning as determined by our computational modelling.

The dependent measure in our analyses was the mean amplitude of the epoch from $500-\mathrm{ms}$ to $800-\mathrm{ms}$ poststimulus onset at frontal and centro-frontal scalp sites (Fp1, Fp2, AF3, AF4, F3, F4, F7, F8, Fz, FC1, FC2, FC5, FC6) for all trials. The independent measure was the model (single-dimension or two-dimension) that provided the better fit for each block of performance. Figure 11 shows the resulting ERP waveforms for each electrode, along with the overall average, and illustrated the late, positive slow wave that emerged in these frontal and central electrodes. The ERP was more positive during blocks that were fit by the single-dimensional rule model (SD) than for blocks fit best by the two-dimensional, conjunctive-rule model (CR).
We then averaged the signals from the 13 electrodes for an overall analysis of variance. A repeated-measures ANOVA confirmed the significant difference between the two mean amplitudes. $F(1,17)=11.00, p<0.004, \eta_{\text {partial }}^{2}=0.393$, reflecting a larger late positive slow wave for trials fit best by the single dimensional model $(M=2.51, S D=3.02)$ compared with trials fit best by the two-dimensional rule model $(M$ $=0.981, S D=2.78$ ). The late positive complex, which is stronger for the single-dimensional blocks, may reflect some aspect of the hypothesis testing procedure or the transition from single- to two-dimensional strategies. This exploratory

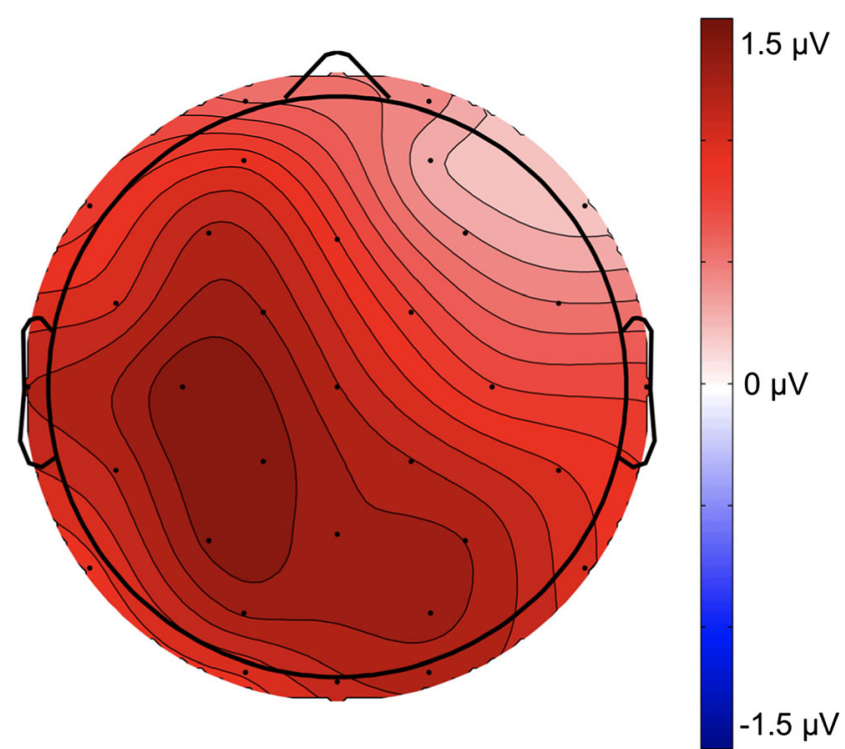

Fig. 10 Topographic maps of hard/easy subtraction (correct responses only) of mean amplitude between 500-800 ms for stimulus-locked ERPs 


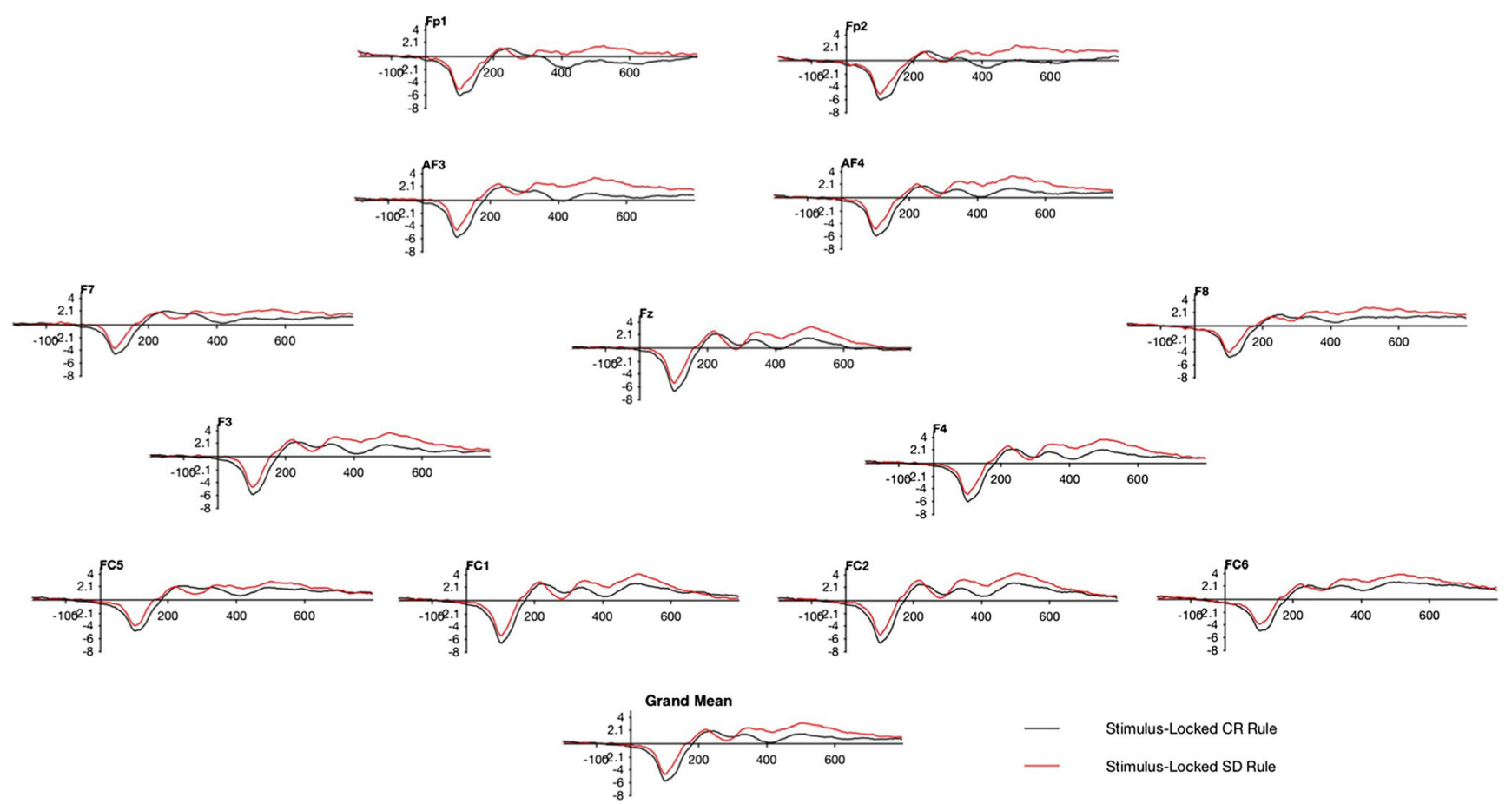

Fig. 11 Mean amplitude between 500-800 ms for stimulus-locked ERPs for trials fit best by the single-dimensional (SD) rule versus trials fit best by the two-dimensional conjunctive rule (CR)

result must be interpreted with caution, and we will discuss this further in the Discussion section.

\section{Discussion}

The primary focus of the present study was to examine if the cognitive processes involved in categorization can be distinguished physiologically. Gabor patch stimuli were used, similar to those used in many behavioural studies involving rulebased category learning. Categorization accuracy was assessed, and stimulus-locked and feedback-locked ERPs were examined. Unlike past studies involving more simple, single-dimensional rule-based categorization tasks, the current study examined how stimuli and feedback are processed when categorization is governed by a more complex rule. Behavioural data indicated that most participants were able to learn the conjunctive rule-based category set, although performance was low during the first few learning blocks as participants tested various rules and changed strategies. Our modelling of the behavioural data revealed that participants varied in terms of which strategy they adopted when completed the conjunctive rule-based task, with the majority of participants adopted the two-dimensional rule-based strategy.

The ERP findings demonstrated that various key components are involved in the categorization process. To begin, when processing the categorization stimuli, an LPC was found, which was larger for correct compared to incorrect trials. This finding is in line with previous research by Morrison et al. (2015), suggesting that an individual's working memory is continually updated when viewing categorization stimuli. As new rules are tested and dismissed, this information is updated in memory. Unlike Morrison et al., who used a single-dimensional rule-based category set, the current study used a conjunctive rule-based category set. One potential difference between these category sets is that in the single-dimensional category set, inhibitory control is required to inhibit one of the stimulus dimensions (e.g., categorize based on the frequency of the lines in the Gabor patch, while ignoring the orientation of the lines), whereas in the conjunctive category set we used, less inhibitory control may be recruited, because participants have to integrate information from both dimensions to arrive at the correct categorization rule (e.g., categorize based on the frequency and orientation of the lines in the Gabor patch). It also is plausible that conjunctive-rule category learning requires substantial inhibition, because the system needs to ignore rules that do not work or were already tried. However, this does not undermine our argument that this category requires less inhibition than a single-dimensional category set. In addition, the conjunctive category set places heavier demands on working memory capacity to solve, because more hypothesis testing is required to arrive at the correct, more complicated rule. The fact that an LPC was found in both a single-dimensional and a conjunctive rule-based category set suggests that this component indexes working memory processes.

Aside from Morrison et al. (2015), who examined the LPC in the context of a category learning task, other studies have supported the idea that the LPC is involved in memory 
updating and decision accuracy. The LPC has been important in studies of explicit recognition memory (Rugg et al., 1998) and is generally found to be largest over parietal scalp sites. Additionally, the LPC also has been shown to be sensitive to decision accuracy. For example, Finnegan, Humphreys, Dennis, and Geffen (2002) found that a larger LPC amplitude was elicited in response to accurately categorized word stimuli. Participants were presented with new unstudied words and old words, which had been presented at an earlier time. Results revealed that LPC amplitude was larger in ERPs evoked by words, which were correctly recognized, compared with incorrect recognition decisions.

Finally, our exploratory analysis also revealed a larger LPC among the strong learners when they engaged in singledimensional learning compared with two-dimensional learning. There seem to be two possible explanations. One possibility is that the higher LPC observed for single-dimensional learning reflects the greater reliance on executive functions at the earlier part of the experiment, when participants were still learning the task and still testing hypotheses. Most of the two-dimensional blocks were recorded later in learning when performance had stabilized. A second possibility is related to the affective nature of the LPC (Schupp et al., 2000). Greater LPC amplitudes have been observed with more positive and more negative stimuli compared with neutral. If the two-dimensional strategy was generally being used later, it is possible that the stimuli were being viewed more neutrally relative to the less pleasant nature of the earlier parts of the task, which featured difficult hypotheses to test and frequent error signals. Given the exploratory nature of the analyses, all of these conclusions are tentative. However, this analysis suggests a new and interesting way to examine category learning by combining the results of computational models with ERP data.

The second cognitive process of interest in the current study was the manner in which participants processed feedback regarding their categorization decisions. More specifically, we were interested in how learners would process negative feedback compared with positive feedback. We found that incorrect trials elicited a more pronounced P300 compared with correct trials, which is in line with research by Morrison et al. (2015). Assuming that learners developed some confidence in their categorization strategy, they would notice the disconnect between a rule-based response and the subsequent unexpected negative feedback. The more pronounced P300 could be an index of that disconnect. The current finding is in line with prior research demonstrating that P300s occur more based on deviant stimuli or stimuli that have lower probabilities (Duncan-Johnson \& Donchin, 1982; Johnson, 1984). Similarly, when presented with a gambling task to complete, Hajcak, Holroyd, Moser, and Simmons (2005) found that P300 amplitude was largest for the unexpected outcomes, confirming that participants indeed formed expectations regarding feedback.
The examination of stimulus-locked and feedback-locked ERPs included data from participants classified as learners. In the current study, learners were classified as a participant adopting either a single-dimensional rule-based strategy or a two-dimensional strategy. While the optimal conjunctive rule would yield the highest accuracy (100\% possible), singledimensional rules based on either dimension could provide an accuracy of up to $80 \%$, and other two-dimensional strategies also could be successful due to the relatively high separability of the four underlying distributions (Zeithamova \& Maddox, 2006). To alleviate any concerns that singledimensional rule learners may be processing the stimuli in a distinctly different manner from two-dimensional rule learners, additional analyses were conducted. Singledimensional rule learners were removed, and the data were reanalyzed only including two-dimensional rule-learners. Results remained significant when excluding singledimensional rule learners from the analysis, suggesting that single-dimensional and two-dimensional strategy users processed the categorization stimuli in a similar manner. That is, both types of strategy users would need to take part in the same cognitive processes (i.e., hypothesis testing, rule switching, and memory updating). Future research would benefit from examining complex rule-based category learning using a category set where only a complicated rule can result in good performance. Adopting a more simplistic rule-based strategy will result in much lower categorization performance. If this were done, good learners and poor learners could be compared to determine whether any differences in processing existed based on the strategy the participant adopted.

In addition to examining stimulus-locked and feedbacklocked ERPs for correct versus incorrect categorization responses, also of interest in the current study was if and how participants would differentially process easy and hard categorization stimuli. Because only correct trials were analyzed, only the strongest learners were included in the analysis to ensure that there was a sufficient number of trials (i.e., at least 30 trials per learner) in each of the category type bins (i.e., A1, A2, A3, and B). Strong learners were classified as any participant who was best fit by a two-dimensional, rule-based strategy during at least the last three learning blocks. Additionally, we were interested in comparing hard versus easy stimuli in strong learners, in particular, because these participants were scoring the highest on the categorization task. Among these high-performers, we were interested in determining whether they would process stimuli differently (easy vs. hard) even though they were categorizing all stimulus types with high optimal rule accuracy. Results revealed a larger late positive slow wave for difficult compared to easy categorization stimuli, confirming that processing differences exist based on stimulus difficulty and taking us a step past what behavioural research can tell us. This is consistent with other research showing that larger, late positive ERPs are associated with 
classifying exemplars that are near the category boundary and thus more difficulty to classify (Folstein \& van Petten, 2011), although they observed this effect primarily in frontal regions, whereas we observed the effect in central parietal region. This difference is not unexpected given the different nature of the category tasks being examined. In general, our study along with other research indicates that behavioural data can show how well participants are performing on a categorization task but tells us very little about stimulus processing demands. This highlights the importance of ERP and computational modelling.

Numerous studies have reported the emergence of positive slow waves (PSW) in more difficult task conditions, unrelated to category learning (Cremer, Kok, Zeef, \& Keuss, 1996; Kok, 1986, 1988; Kok, Vijver, \& Rooijakkers, 1985). For example, research by Ruchkin (Ruchkin et al., 1988; Ruchkin \& Sutton, 1983) showed that PSWs with a centroparietal distribution were elicited by target stimuli in difficult perceptual discrimination tasks. These findings are in line with results from the current study, which found a PSW for more difficult task stimuli in a perceptual-based category learning task across centroparietal electrodes. Various explanations have been given to explain PSWs. Ruchkin et al. (1988) suggested that PSWs reflect the difficulty of perceptual operations and memory storage. A second suggestion has been that PSWs are functionally closely related to $\mathrm{P} 3$ and represent continued processing of perceptually difficult stimuli (Kok \& Looren de Jong, 1980; Ruchkin, Sutton, Kietzman, \& Silver, 1980). These prior findings support the PSW found in the present study, because our more difficult categorization stimuli (i.e., A2 \& A3) were perceptually similar to the opposing Category type (i.e., B) and furthermore required heavier processing efforts to retrieve information about these categorization stimuli from memory. Future research may benefit from examining whether PSWs are a functionally distinct ERP component related to task difficulty or whether PSWs represent a delayed P3. Furthermore, the emergence of PSWs for difficult categorization stimuli has important implications for our understanding of complex, rule-based category learning. In everyday life, we often encounter various members of a category, some more difficult than others to categorize. Given the fact that not all members of a category set are treated/processed in the same way, ERP research on category learning can shed light on how stimulus difficulty impacts the categorization process.

In summary, the current study took a novel approach to understanding the cognitive mechanisms involved in making categorization decisions by measuring event-related potentials during a complex rule-based task. Overall, findings demonstrated the effectiveness of real-time neural monitoring during category learning and provide evidence highlighting the cognitive mechanisms involved in rule-based category learning. Results suggested that learning complex categories engenders qualitative changes in brain activity that are marked by a late positive component during stimulus processing, indexing the updating process of working memory. Additionally, a P300 component was present during feedback processing, indexing confidence in categorization decisions and showing the different neural responses to correct and incorrect trials. An analysis of the differential responding to difficult and less difficult stimuli revealed a larger late positive slow wave for more difficult stimuli. This finding suggests that all categorization stimuli are not treated/processed the same way, even if performance is quite high. The ERP analyses clearly allowed us to understand the nature of how these different kinds of stimuli were processed in a way that traditional behavioral analyses could not. This suggests that ERP analyses can be used to better understand the cognitive processes involved in different categorization tasks. Future research should consider not only ERP measurements but also should combine those measurements with insights from computational models, which can help to connect brain electrophysiology with the cognitive psychology of these fundamental tasks.

Acknowledgements This research was supported by research grants from the Natural Sciences and Engineering Research Council (NSERC) of Canada to MFJ and JPM and an NSERC Canada Graduate Scholarship to RR. Infrastructure support was provided by an NSERC RTI grant. The authors thank Kazunaga Matsuki for his development and assistance of the strategy analysis program.

\section{References}

Ashby, F. G. (1992). Multidimensional Models of Perception and Cognition. L. Erlbaum.

Ashby, F. G., \& Ennis, J. M. (2006). The role of the basal ganglia in category learning. In Psychology of Learning and Motivation Volume 46 (Vol. 46, pp. 1-36). Elsevier.

Ashby, F. G., \& O’Brien, J. B. (2005). Category learning and multiple memory systems. Trends in Cognitive Sciences, 9(2), 83-89.

Cincotta, C. M., \& Seger, C. A. (2007). Dissociation between striatal regions while learning to categorize via feedback and via observation. Journal of Cognitive Neuroscience, 19(2), 249-265.

Craddock, R. C., Milham, M. P., \& LaConte, S. M. (2013). Predicting intrinsic brain activity. NeuroImage, 82, 127-136.

Cremer, R., Kok, A., Zeef, E., \& Keuss, P. (1996). Age-related effects of different types of noise and stimulus quality: An event-related potential (ERP) study. Journal of Psychophysiology, 10, 239-251.

Delorme, A., \& Makeig, S. (2004). EEGLAB: An open source toolbox for analysis of single-trial EEG dynamics including independent component analysis. Journal of Neuroscience Methods, 134(1), 921.

Dien, J., Spencer, K. M., \& Donchin, E. (2004). Parsing the late positive complex: Mental chronometry and the ERP components that inhabit the neighborhood of the P300. Psychophysiology, 41(5), 665-678.

Duncan-Johnson, C. C., \& Donchin, E. (1982). The P300 component of the event-related brain potential as an index of information processing. Biological Psychology, 14(1-2), 1-52.

Filoteo, J. V., Lauritzen, S., \& Maddox, W. T. (2010). Removing the frontal lobes: The effects of engaging executive functions on perceptual category learning. Psychological Science, 21(3), 415-423.

Filoteo, J. V., Maddox, W. T., Simmons, A. N., Ing, A. D., Cagigas, X. E., Matthews, S., \& Paulus, M. P. (2005). Cortical and subcortical brain 
regions involved in rule-based category learning. Neuroreport, 16(2), 111-115.

Folstein, J. R., \& van Petten, C. (2011). After the P3: Late executive processes in stimulus categorization. Psychophysiology, 48(6), 825-841.

Grossman, M., Koenig, P., DeVita, C., Glosser, G., Alsop, D., Detre, J., \& Gee, J. (2002). The neural basis for category-specific knowledge: An fMRI study. NeuroImage, 15(4), 936-948.

Gunter, T. C., Jackson, J. L., \& Mulder, G. (1995). Language, memory, and aging: An electrophysiological exploration of the N400 during reading of memory-demanding sentences. Psychophysiology, 32, $215-225$.

Hajcak, G., Holroyd, C. B., Moser, J. S., \& Simons, R. F. (2005). Brain potentials associated with expected and unexpected good and bad outcomes. Psychophysiology, 42(2), 161-170.

Haruno, M., \& Kawato, M. (2006). Heterarchical reinforcement-learning model for integration of multiple cortico-striatal loops: fMRI examination in stimulus-action-reward association learning. Neural Networks: The Official Journal of the International Neural Network Society, 19(8), 1242-1254.

Huang-Pollock, C. L., Maddox, W. T., \& Karalunas, S. L. (2011). Development of implicit and explicit category learning. Journal of Experimental Child Psychology, 109(3), 321-335.

Jiang, X., Bradley, E., Rini, R. A., Zeffiro, T., Vanmeter, J., \& Riesenhuber, M. (2007). Categorization training results in shapeand category-selective human neural plasticity. Neuron, 53(6), 891-903.

Johnson, R., Jr. (1984). P300: A model of the variables controlling its amplitude. Annals of the New York Academy of Sciences, 425, 223-229.

Kok, A. (1986/8). Effects of degradation of visual stimuli on components of the event-related potential (ERP) in go/nogo reaction tasks. Biological Psychology, 23(1), 21-38.

Kok, A. (2001). On the utility of P3 amplitude as a measure of processing capacity. Psychophysiology, 38(3), 557-577.

Kok, A., \& Looren de Jong, H. (1980). Components of the event-related potential following degraded and undegraded visual stimuli. Biological Psychology, 11(2), 117-133.

Kok, A., Vijver, F. R., \& Rooijakkers, J. (1985). Effects of Visual Field, Stimulus Degradation, and Level of Practice on Event-Related Potentials of the Brain. Psychophysiology, 22(6), 707-717.

Li, S., Mayhew, S. D., \& Kourtzi, Z. (2009). Learning shapes the representation of behavioral choice in the human brain. Neuron, 62(3), 441-452.

Lopez-Calderon, J., \& Luck, S. J. (2014). ERPLAB: An open-source toolbox for the analysis of event-related potentials. Frontiers in Human Neuroscience, 8, 213.

Maddox, W. T., \& Ashby, F. G. (1993). Comparing decision bound and exemplar models of categorization. Perception \& Psychophysics, 53(1), 49-70.

Maddox, W. T., Pacheco, J., Reeves, M., Zhu, B., \& Schnyer, D. M. (2010). Rule-based and information-integration category learning in normal aging. Neuropsychologia, 48(10), 2998-3008.

Merchant, H., Zainos, A., Hernández, A., Salinas, E., \& Romo, R. (1997). Functional properties of primate putamen neurons during the categorization of tactile stimuli. Journal of Neurophysiology, 77(3), 1132-1154

Miles, S. J., \& Minda, J. P. (2011). The effects of concurrent verbal and visual tasks on category learning. Journal of Experimental Psychology. Learning, Memory, and Cognition, 37(3), 588-607.

Minda, J. P., Desroches, A. S., \& Church, B. A. (2008). Learning ruledescribed and non-rule-described categories: A comparison of children and adults. Journal of Experimental Psychology. Learning, Memory, and Cognition, 34(6), 1518-1533.
Minda, J. P., \& Rabi, R. (2015). Ego depletion interferes with rule-defined category learning but not non-rule-defined category learning. Frontiers in Psychology, 6, 35.

Monchi, O., Petrides, M., Petre, V., Worsley, K., \& Dagher, A. (2001). Wisconsin Card Sorting revisited: Distinct neural circuits participating in different stages of the task identified by event-related functional magnetic resonance imaging. The Journal of Neuroscience: The Official Journal of the Society for Neuroscience, 21(19), 77337741

Morrison, R. G., Reber, P. J., Bharani, K. L., \& Paller, K. A. (2015). Dissociation of category-learning systems via brain potentials. Frontiers in Human Neuroscience, 9, 389.

Nadler, R. T., Rabi, R., \& Minda, J. P. (2010). Better mood and better performance learning rule-described categories is enhanced by positive mood. Psychological Science, 21(12), 1770-1776.

Nomura, E. M., \& Reber, P. J. (2008). A review of medial temporal lobe and caudate contributions to visual category learning. Neuroscience and Biobehavioral Reviews, 32(2), 279-291.

Poldrack, R. A., \& Foerde, K. (2008). Category learning and the memory systems debate. Neuroscience and Biobehavioral Reviews, 32(2), 197-205.

Polich, J. (2007). Updating P300: An integrative theory of P3a and P3b. Clinical Neurophysiology: Official Journal of the International Federation of Clinical Neurophysiology, 118(10), 2128-2148.

Psychology Software Tools, I. (2012). E-Prime 2.0 (Version 2). Pittsburgh, PA. Retrieved from http://www.pstnet.com

Rabi, R., Miles, S. J., \& Minda, J. P. (2015). Learning categories via rules and similarity: Comparing adults and children. Journal of Experimental Child Psychology, 131, 149-169.

Rabi, R., \& Minda, J. P. (2014). Rule-based category learning in children: The role of age and executive functioning. PloS One, 9(1), e85316.

Rabi, R., \& Minda, J. P. (2016). Category learning in older adulthood: A study of the Shepard, Hovland, and Jenkins (1961) tasks. Psychology and Aging, 31(2), 185-197.

Rabi, R., \& Minda, J. P. (2017). Familiarization may minimize agerelated declines in rule-based category learning. Psychology and Aging, 32(7), 654-674.

Ruchkin, D. S., Johnson, R., Jr, Mahaffey, D., \& Sutton, S. (1988). Toward a functional categorization of slow waves. Psychophysiology, 25(3), 339-353.

Ruchkin, D. S., \& Sutton, S. (1983). 11 Positive Slow Wave and P300: Association and Disassociation. Advances in Psychology, 10, 233 250.

Ruchkin, D. S., Sutton, S., Kietzman, M. L., \& Silver, K. (1980). Slow wave and P300 in signal detection. Electroencephalography and Clinical Neurophysiology, 50(1-2), 35-47.

Rugg, M. D., Mark, R. E., Walla, P., Schloerscheidt, A. M., Birch, C. S., \& Allan, K. (1998). Dissociation of the neural correlates of implicit and explicit memory. Nature, 392(6676), 595-598.

Rushby, J. A., \& Barry, R. J. (2007). Event-related potential correlates of phasic and tonic measures of the orienting reflex. Biological Psychology, 75(3), 248-259.

Schendan, H. E., \& Lucia, L. C. (2010). Object-sensitive activity reflects earlier perceptual and later cognitive processing of visual objects between 95 and 500 ms. Brain Research, 1329, 124-141.

Schnyer, D. M., Maddox, W. T., Ell, S., Davis, S., Pacheco, J., \& Verfaellie, M. (2009). Prefrontal contributions to rule-based and information-integration category learning. Neuropsychologia, 47(13), 2995-3006.

Schupp, H. T., Cuthbert, B. N., Bradley, M. M., Cacioppo, J. T., Ito, T., \& Lang, P. J. (2000). Affective picture processing: The late positive potential is modulated by motivational relevance. Psychophysiology, $37(2), 257-261$.

Seger, C. A. (2008). How do the basal ganglia contribute to categorization? Their roles in generalization, response selection, and learning 
via feedback. Neuroscience and Biobehavioral Reviews, 32(2), 265-278.

Seger, C. A., \& Miller, E. K. (2010). Category learning in the brain. Annual Review of Neuroscience, 33, 203-219.

Smith, E. E., \& Grossman, M. (2008). Multiple systems of category learning. Neuroscience and Biobehavioral Reviews, 32(2), 249264.

Smith, E. E., Patalano, A. L., \& Jonides, J. (1998). Alternative strategies of categorization. Cognition, 65(2-3), 167-196.
Waldron, E. M., \& Ashby, F. G. (2001). The effects of concurrent task interference on category learning: Evidence for multiple category learning systems. Psychonomic Bulletin \& Review, 8(1), 168-176.

Williams, Z. M., \& Eskandar, E. N. (2006). Selective enhancement of associative learning by microstimulation of the anterior caudate. Nature Neuroscience, 9(4), 562-568.

Zeithamova, D., \& Maddox, W. T. (2006). Dual-task interference in perceptual category learning. Memory \& Cognition, 34(2), 387-398. 